\title{
Neutron-Insensitive Gamma-Ray Detector with Aerogel for Rare Neutral-Kaon Decay Experiment
}

\author{
Yosuke Maeda* \\ Kyoto University \\ E-mail: maeda_ydscphys.kyoto-u.ac.jp
}

A novel $\gamma$-ray detector insensitive to neutrons is developed to study the CP-violating rare decay $K_{\mathrm{L}} \rightarrow \pi^{0} v \bar{v}$ at a $10^{-11}$ sensitivity by the J-PARC KOTO experiment. By using aerogel as Cerenkov radiator, both an excellent detection efficiency to photons and blindness to neutrons are achieved. A Monte Carlo simulation including ray tracing of Čerenkov light was developed. It reproduced well the detector response of a real-size prototype in a beam test. The detector was partially installed as an in-beam photon veto detector for the KOTO first physics data taking in May 2013. Its stability and performance in the real beam environment were evaluated. The detector was found to meet the design specifications and to be usable for physics data analysis.

Technology and Instrumentation in Particle Physics 2014,

2-6 June, 2014

Amsterdam, the Netherlands

\footnotetext{
* Speaker.
} 


\section{The $K_{\mathrm{L}} \rightarrow \pi^{0} v \bar{v}$ Decay and the KOTO Experiment}

The matter-antimatter asymmetry in the universe is one of the most outstanding problems in modern physics. From the view point of elementary particle physics, the search for new sources of CP-violation plays an important role in understanding this issue. The CP-violating rare decay $K_{\mathrm{L}} \rightarrow \pi^{0} v \bar{v}$ is an excellent probe of new physics due to the strongly suppressed Standard Model (SM) contribution and its theoretical cleanness.

The KOTO ( $\mathrm{K}^{0}$ at TOkai) experiment[四] is dedicated to study the $K_{\mathrm{L}} \rightarrow \pi^{0} v \bar{v}$ at the Japan Proton Accelerator Research Complex (J-PARC). Its goal is the first observation of the decay by achieving a $10^{-11}$ sensitivity, comparable to the SM branching ratio prediction of $(2.4 \pm 0.4) \times$ $10^{-11}[$ [ $]$. This represents a three order-of-magnitude improvement from the current direct upper limit of $2.6 \times 10^{-8}$ at $90 \%$ C.L. achieved by the KEK E391a experiment[[3].

\section{Experimental Method and Detector Requirements}

In the KOTO experiment, a narrowly-collimated secondary neutral beam[田] is used as a source of $K_{\mathrm{L}}$. Such beam is generated from bombarding the production target with an intense $30 \mathrm{GeV}$ primary proton beam from the J-PARC Main Ring[[]]. Since the only observable particles in the $K_{\mathrm{L}} \rightarrow \pi^{0} v \bar{v}$ decay are 2 photons from the $\pi^{0}$, signal events are identified by detecting $2 \gamma$ shower with an electromagnetic calorimeter and "nothing" else with veto detectors surrounding the decay region. Any inefficiencies in the veto detectors result in events with the same final topology and hence produce background. Thus, the performance of the veto detectors is a key in the search for this decay.

To achieve higher sensitivities, the use of an intense $K_{\mathrm{L}}$ beam and an hermetic background rejection is essential. $K_{\mathrm{L}} \rightarrow 2 \pi^{0}$ decay with one $\gamma$ escaping in the forward direction through the calorimeter beam hole is one of the most serious background sources. The Beam Hole Photon Veto detector (BHPV) is designed to detect such $\gamma^{\prime}$ s. Its inefficiency is required $10^{-3}$ for high energy $\gamma(>1 \mathrm{GeV})$ to reduce such background to less than the SM $K_{\mathrm{L}} \rightarrow \pi^{0} v \bar{v}$ prediction. On the other hand, the neutral beam escaping through the calorimeter beam hole contains not only $K_{\mathrm{L}}$ but also numerous neutrons and low energy $\gamma^{\prime}$ s. Hence the BHPV must be insensitive to these neutrons while keeping high detection efficiency to $\gamma$ from $K_{\mathrm{L}}$ decay. The neutron flux is estimated to be $0.5 \mathrm{GHz}$ for the beam with design power. A conventional $\gamma$ detector, such as calorimeter, is not a viable solution with respect to counting rates and signal losses. To overcome this situation, a novel $\gamma$ detector utilizing C̆erenkov light from aerogel was developed.

\section{Detector Design}

The detector consists of 25 modules, each of which contains a lead sheet, a block of silica aerogel, flat mirrors, Winston Cone[6] light collection funnels and two 5-inch PMTs as shown in Figure $\mathrm{W}$. Incident $\gamma^{\prime} \mathrm{s}$ are converted into electron-positron pairs and the Čerenkov lights generated during their passage in the aerogel are detected. When neutrons hit the detector, protons and pions are mainly produced due to scatterings or hadronic interactions. By choosing the refractive index of the aerogel to be 1.03 , no or little Čerenkov lights are emitted by such particles thanks to their small 
velocity compared with C̆erenkov threshold, while enough light yield is obtained for electrons or positrons. In this way, this detector is insensitive to neutrons but keeps excellent detection efficiency for $\gamma^{\prime}$ s.

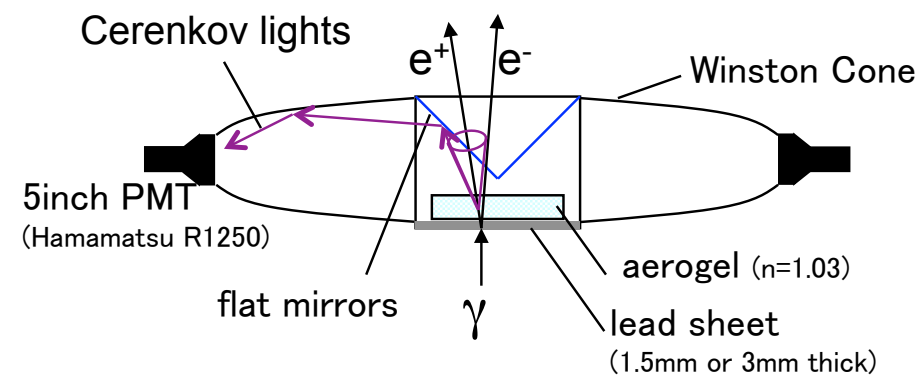

Figure 1: Schematic drawing of the detector module.

A Winston Cone funnel is used to optimize light collection. This has a shape of an off-axis parabola of revolution, and has $100 \%$ light collection efficiency for photons with smaller incident angle than its critical angle $\theta_{\mathrm{c}} . \theta_{\mathrm{c}}$ is defined as the ratio of the entrance and exit diameters $T_{\mathrm{in}}$ and $T_{\text {out }}$ of the cone:

$$
\sin \theta_{\mathrm{c}}=\frac{T_{\text {out }}}{T_{\text {in }}}
$$

$T_{\text {in }}$ and $T_{\text {out }}$ are determined by the size of the beam and that of PMTs respectively and give $\theta_{\mathrm{c}}=$ $23.6^{\circ}$. Since the Cerenkov angle for aerogel with $n=1.03$ is $13.9^{\circ}$, smaller than the Winston Cone critical angle, it is possible to collect the Čerenkov lights effectively.

The coincidence of 3 or more sequential modules is used to further separate neutrons and low energy $\gamma^{\prime}$ s form the relatively high energy $\gamma^{\prime}$ s generated by $K_{\mathrm{L}}$ decay products. This is because hadronic showers induced by neutrons tend to develop isotropically, while electromagnetic showers induced by $\gamma^{\prime}$ s tend to develop in the forward direction. On the other hand, the length of the electromagnetic showers depends on the energy of the $\gamma$. As a consequence, neutrons and low energy $\gamma^{\prime}$ s are not easily detected by a coincidence over multiple modules.

\section{Monte Carlo Simulation}

An optical photon tracing code was developed to understand the detector response using a Monte Carlo (MC) simulation The generation of particle showers and Čerenkov light is made by Geant $4[$ []]. The information on the position, direction and wavelength for the generated Čerenkov radiation is used to simulate the transportation of the photon from the aerogel to the photo cathode of the photo multiplier tube (PMT) via flat mirrors and Winston Cone.

The effects of absorption and Rayleigh scattering, which are wavelength independent and dependent respectively, are considered for the photon tracing in the aerogel. For a given photon wavelength $\lambda$, the probability of absorption and scattering per unit length is described as $A$ and $\frac{C}{\lambda^{4}}$, respectively. The parameters $A$ and $C$ are derived from the direct light transmission measurement using the setup as shown in Figure $\square$. The light output was measured by 2 PMTs, where one of them is for reference and the measured output is always normalized to the measurement of this PMT. The 
ratio with the PMT readings with aerogel inserted to and removed from the space between the LED and PMT was defined as transmission for a given aerogel at the LED wavelength, and the results were fitted with the following Hunt formula :

$$
A \exp \left(-\frac{C}{\lambda^{4}}\right)
$$

Figure [3] shows an example of the fit. The wavelength dependence is well described by formula (4.]). The average fit value over multiple aerogel samples was implemented into the simulation code. The ray tracing is terminated when the photon is absorbed by aerogel and the direction is changed by an angle $\theta$, randomly drawn by a $\left(1+\cos ^{2} \theta\right)$ distribution.

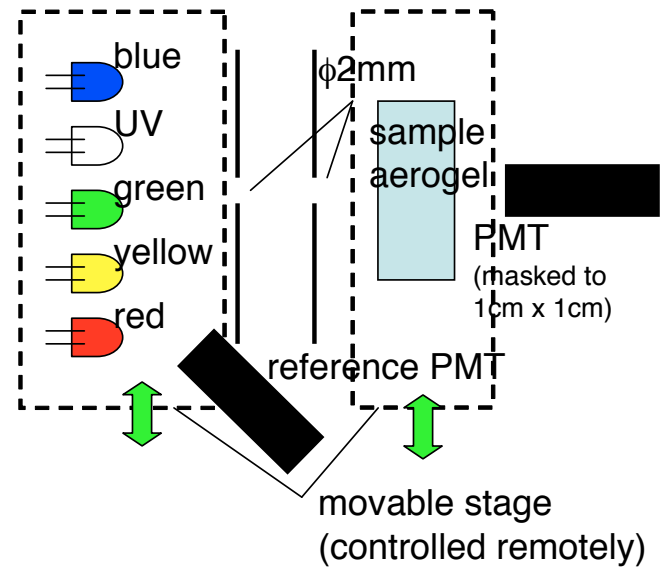

Figure 2: Setup for the aerogel transmission measurement.

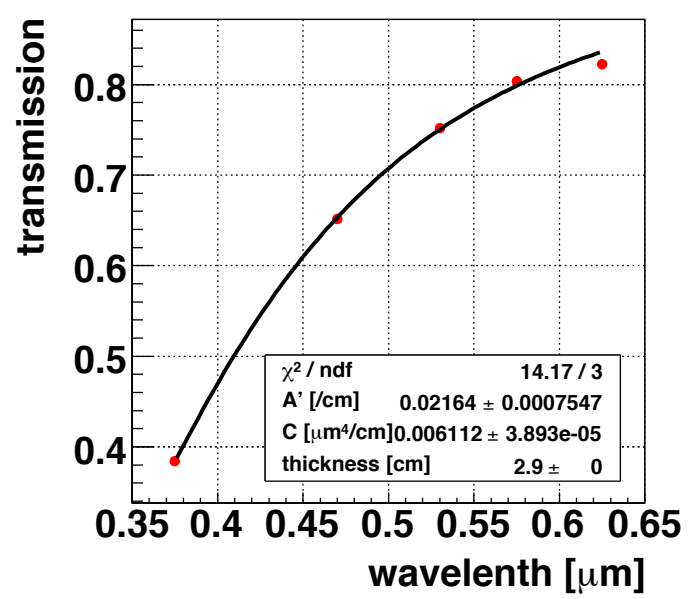

Figure 3: Result of transmission measurement.

Photons escaping the aerogel are undergo reflection in the flat mirrors and Winston Cone before being collected by the photocathod of the PMT. The reflection efficiency of mirror and Winston Cone is assumed to be $85 \%$ regardless of the photon wavelength. Finally, the photon detection efficiency is modeled using the quantum efficiency of the PMT as per data sheet specifications.

\section{Performance Test with Positron Beam}

To check the validity of the MC simulation described in the previous section, the performance of the detector was tested with a full-size prototype irradiated with a $600 \mathrm{MeV} / c$ positron beam in the Laboratory of Nuclear Science (presently Research Center for Electron Photon Science) at Tohoku University, Japan.

The incident beam was triggered by 2 plastic scintillation counters of $1 \mathrm{~cm} \times 1 \mathrm{~cm}$ cross section, located upstream and downstream of the prototype detector. The position dependence of the light yield was studied by moving the detector with respect to the beam. The output of each PMT was converted in the photoelectron(p.e.) unit by using an LED calibration, and compared to the MC expectations. The result is shown in Figure . Although the absolute light yield was 55\% of the simulation prediction, the position dependence is well reproduced by the scaled MC output. Deterioration of the aerogel surface during its transportation to the facility is thought to be a reason of the light yield discrepancy. The friction between aerogel tiles makes their surface coarser. 
Additional photon absorption or scattering can occur on this damaged surface. The dependence of the PMT quantum efficiency on the incident angle can also contribute to the discrepancy due to the polarization of the Čerenkov radiation. A more precise modeling of photon the detection considering these effect has not been attempted.

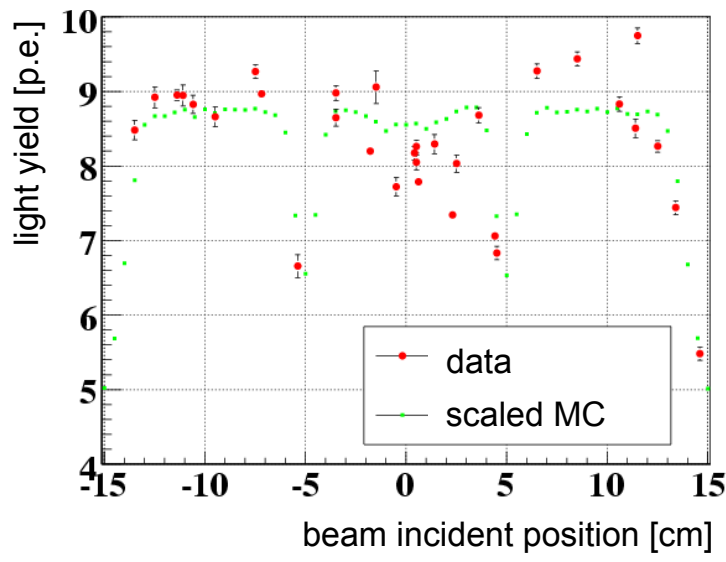

Figure 4: Measured position dependence with a $600 \mathrm{MeV} / c$ positron beam for the full-size prototype detector. $0 \mathrm{~cm}$ means the center of the module.

\section{Performance during the Physics Data Taking}

Twelve modules out of the 25 in the final BHPV configuration were installed in the experimental area during the KOTO first physics data taking in May 2013 and used as a veto detector.

\subsection{Stability in the Beam Environment}

The stability during the one month beam operations was studied by using LEDs equipped with each module and penetrating charged particles tagged by upstream and downstream plastic scintillators in the dedicated calibration runs.

The PMT gain stability was checked by the LED data. The single photoelectron peak was monitored and used for the p.e. calibration. An example of the variation during the run is shown in Figure [1, the single p.e. gain was stable within $\pm 5 \%$. The gain deviation between beam-on and beam-off was evaluated by comparing the LED light yield. Since the contribution of accidental hits is not negligible in the beam-on data, it was is subtracted using data from random triggers collected without the LEDs flashing. The time dependence of the ratio is shown in Figure 6. It shows that the gain was unchanged within $\pm 5 \%$ through the data taking period.

The light yield of each aerogel was monitored every 1 or 2 days by a special run, during which a piece of brass was inserted in the beam line. In this condition, the detectors counting rate was drastically reduced, while charged particles from the $K_{\mathrm{L}}$ decays can be easily tagged by requiring the coincidence of plastic scintillators located upstream and downstream of the BHPV detector. In addition, to remove the velocity dependence of the light yield, hits in the all other modules are required. Figure $\square$ shows an example of the light yield distribution; a clear peak was observed around 6-9 p.e. The history of the peak value during the data taking period is shown in 


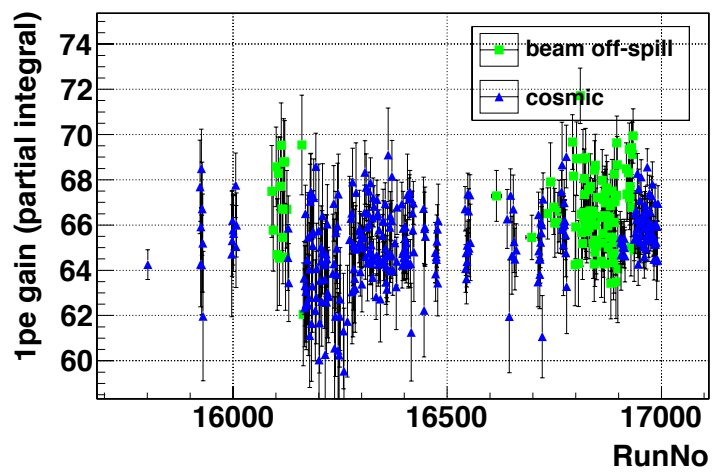

Figure 5: Example of the history of 1 p.e. gain. Green(blue) means that the data was obtained during the beam(cosmic) data taking period.

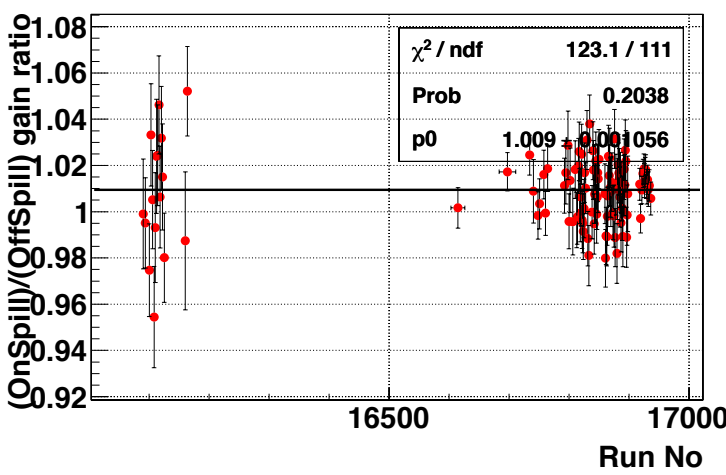

Figure 6: Ratio of the LED outputs between beamon and beam-off periods.

Figure 8 . Although there is a module-by-module variation in the absolute light yield value, the stability of the central value for each module is within $4 \%$ throughout the beam operations. The MC simulation is tuned to reproduce this light yield. The module-by-module difference was also taken into consideration.

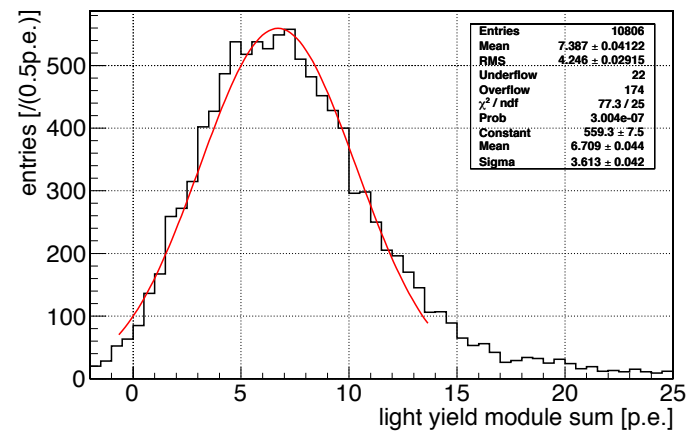

Figure 7: Example of aerogel light yield distribution for penetrating charged particles.

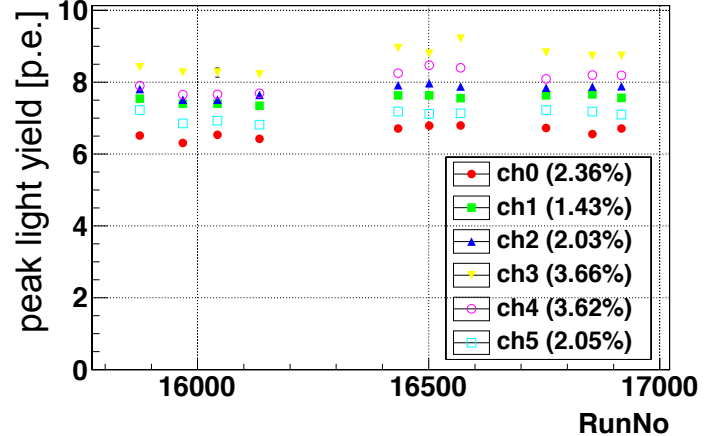

Figure 8: History of aerogel light yield during the beam operation for the upstream six modules. Fit peak values (as in Figure 7) for each module are plotted. Values in parenthesis in the legend are the standard deviation divided by the mean through the data taking period.

\subsection{Counting Rate}

The counting rate during beam operation was evaluated using a $500 \mathrm{MHz}$ FADC[ [ $]$. The number of pulses larger than 1.5 p.e. was counted in random trigger events. The results are shown in Figure 8 together with MC expectations. The measured rate is below $2 \mathrm{MHz}$, which is within a tolerable level. In MC, the dominant contribution is not from neutrons but beam $\gamma^{\prime}$ s, which confirms excellent blindness to neutrons. The agreement between data and $\mathrm{MC}$, although within a factor of 2 , is reasonable if one considers the uncertainty in the MC prediction for the beam particle contents. 


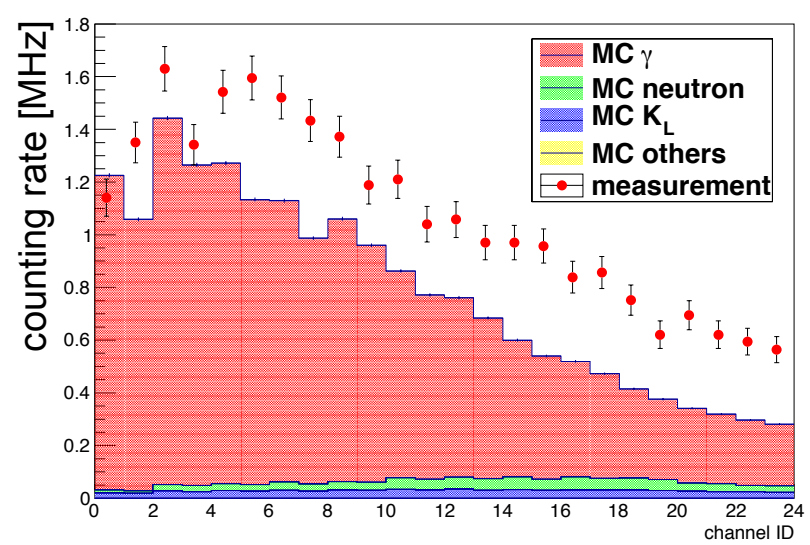

Figure 9: Counting rate for each channel in random beam events. Smaller channel ID is for upstream module. Channels with neighboring even and odd ID belongs to the same module. The histograms for MC are stacked on one another; thus the histogram for "MC $\gamma$ " is the sum of all the components.

\subsection{Response to $\gamma$ from $K_{\mathrm{L}}$ Decay}

The response to $\gamma$ was studied by using photons from $K_{\mathrm{L}}$ decays. $K_{\mathrm{L}} \rightarrow 3 \pi^{0}$ events are tagged by requiring 5 clusters in the central electromagnetic calorimeter. Assuming that the $K_{\mathrm{L}}$ decays on the beam axis without transverse momentum, its invariant mass can be reconstructed from the energy and position information of the 5 clusters. The mass distribution is shown in Figure $\mathbb{}$. A clear peak was observed at the $K_{\mathrm{L}}$ mass in both data and MC. Both distributions agreed well, which means quite clean $K_{\mathrm{L}} \rightarrow 3 \pi^{0}$ sample was obtained. Figure $\mathbb{}$ l] shows the timing distribution of BHPV hits with above selection superimposed to events with 6 clusters. In the 6-cluster sample, we expect no activity associated with a real $\gamma$ from $K_{\mathrm{L}} \rightarrow 3 \pi^{0}$ decay. The periodic distribution seen in these accidental hits reflects the beam bunch structure. In the 5-cluster selection, there should be a remaining $\gamma$, which sometimes goes to the forward direction and hits the BHPV. A sharp peak is observed in the timing distribution for the 5-cluster sample and this was a clear evidence that such $\gamma$ was successfully detected by BHPV.

For further understanding of this detector, various distributions were compared between data and $\mathrm{MC}$ for events with 4 clusters in the calorimeter. Figure $\mathbb{1 2}$ and $\mathbb{1 3}$ show comparison of the distributions for the most upstream channel ID and light yield sum over all modules with coincidence hits. The contribution from accidental hits was estimated with the 6-cluster selection data and subtracted from the distribution. The nice agreement confirms that the performance of this detector in the data taking conditions is well understood.

\section{Summary and Prospects}

A novel $\gamma$ detector using aerogel was developed for the beam hole photon veto detector in the KOTO experiment. The detector achieved both excellent $\gamma$ detection efficiency and blindness to neutrons. The performance was well described by MC simulation both in the beam test and the physics data taking in May, 2013. 


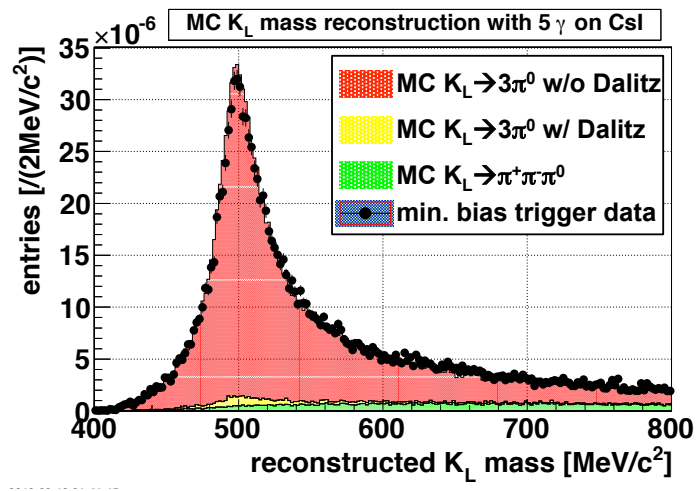

Figure 10: Reconstructed $K_{\mathrm{L}}$ mass distribution in 5-cluster events. Distributions of data and MC are normalized to the same number of entries. In the reconstruction process, 2 solutions appear and the one closer to the $K_{\mathrm{L}}$ mass is chosen. In referring to "5cluster selection" except for this distribution, one solution is required within $\pm 30 \mathrm{MeV} / c^{2}$ from the $K_{\mathrm{L}}$ mass and the other not within $\pm 50 \mathrm{MeV} / c^{2}$.

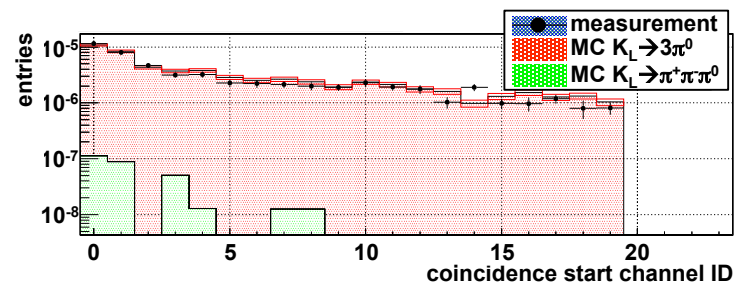

Figure 12: Distribution of the most upstream channel ID among coincidence modules in 4-cluster sample.

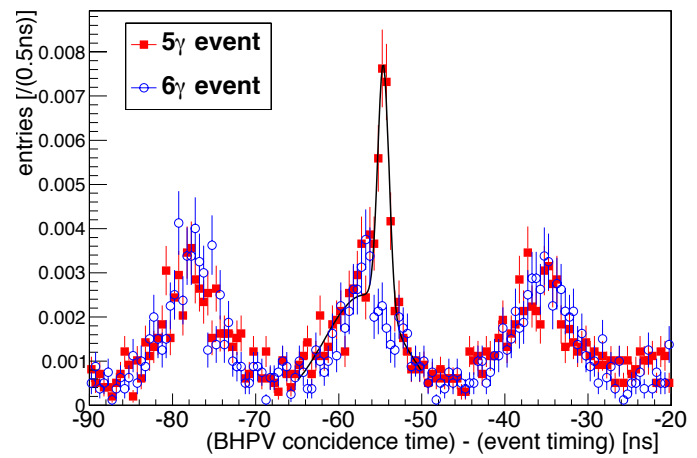

Figure 11: Timing distribution of BHPV with respect to the vertex time with time-of-flight correction in 5-cluster(red) and 6-cluster(blue) events. For BHPV, coincidence of 3 or more successive modules is required as described in Section 3, and the average coincidence time is used. The distribution for the 5cluster selection is fitted with a double gaussian. $\sigma$ of the sharper peak, deemed to be due to real $K_{\mathrm{L}}$ events, is $0.7 \mathrm{~ns}$.

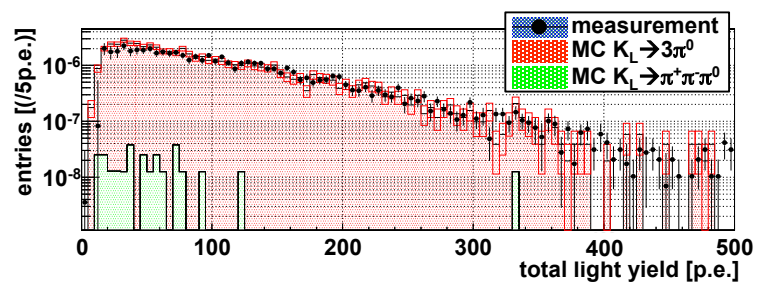

Figure 13: Distribution of total light yield for data and MC simulation in 4-cluster sample.

Only half number of modules are currently installed for the physics data taking, which was not enough to achieve the design goal or the KOTO sensitivity. The full detector construction and installation will be done in steps, according to the sensitivity in each stage.

\section{References}

[1] T. Yamanaka, Prog. Theor. Exp. Phys. (2012) 02B006.

[2] J. Brod, M. Gorbahn and E. Stamou, Phys. Rev. D83, 034030 (2011).

[3] J. K. Ahn et. al., Phys. Rev. D81, 072004 (2010).

[4] T. Shimogawa et. al., Nucl. Instr. and Meth. A 623 (2010) 585.

[5] T. Koseki et. al., Prog. Theor. Exp. Phys. (2012) $02 B 004$.

[6] Winston R., J. Opt. Soc. Amer., 60, 245 (2004).

[7] S. Agostinelli, et al., Nucl. Instr. and Meth. A 506 (2003) 250.

[8] Bogdan, M.; Genat, J. -F; Yau Wah, Real Time Conference (RT), 2010 17th IEEE-NPSS , vol., no., pp.1,2, 24-28 May 2010 